Grenzen weiterdenken 


\title{
Grenze als Methode oder die Vervielfältigung der Arbeit
}

\author{
Sandro Mezzadra und Brett Neilson
}

\begin{abstract}
Der Beitrag enthält Auszüge des Buchs Border as Method, or, the Multiplication of Labor und eine kurze Einordnung. Der erste Teil beschreibt den konzeptuellen Rahmen: Grenzen sind demnach nicht nur als Forschungsobjekt wahrzunehmen, sondern auch als epistemologische Perspektive. Entsprechend werden sowohl die komplexen Besonderheiten von Grenze als auch deren „weltkonfigurierende Funktion“ (Balibar) diskutiert. Der zweite Teil des Beitrags betrachtet Grenze aus Sicht einer Kritik der politischen Ökonomie. Die Beziehungen zwischen Grenze und Arbeit werden kritisch beleuchtet, um die ,Vervielfältigung der Arbeit` zu bestimmen, welche die traditionelle Sichtweise der internationalen Arbeitsteilung aufgreift sowie verkompliziert.
\end{abstract}

\section{Schlagwörter}

Grenze, Methode, internationale Arbeitsteilung, Kritik der politischen Ökonomie der Grenze, Arbeitskraft

\section{Einleitung}

Der folgende Text umfasst zwei Abschnitte aus Kapitel eins (The Proliferation of Borders) sowie zwei Abschnitte aus Kapitel drei (Frontiers of Capital) unserer Publikation Border as Method, or, the Multiplication of Labor (Mezzadra/Neilson 2013). Während der erste Teil des Textes den konzeptuellen Rahmen unserer Untersuchungen beschreibt, veranschaulicht der zweite, welche Entwicklungsmöglichkeiten unser Ansatz birgt, Grenze als Methode im Sinne einer Kritik der politischen Ökonomie zu begreifen. Eine der wesentlichen Ideen des Buches zielt schlussendlich darauf ab, die Themenkomplexe Kapital und Arbeit aus der Perspektive der Grenze zu erfassen und damit eines der wesentlichen Merkmale unseres Ansatzes innerhalb der (kritischen) Grenzforschung auf den Punkt zu bringen. Die zunehmende Gewalt an Grenzen in vielen Teilen der Welt, die sich üblicherweise gegen Migrierende und Asylsuchende richtet, hat in den letzten Jahren zu einer deutlichen Zunahme an wissenschaftlichen Untersuchungen geführt, die sich hauptsächlich auf die Kritik an den politischen und rechtlichen Aspekten der Grenzregime beschränkten. Begriffe wie Staatsgewalt, Ausnahmezustand, Bürgerrechte und Menschenrechte stehen an der Grenze zur Debatte. Dies ist auch Thema in Kapitel sechs unseres Buches, The Souvereign Machine of Governmentality. Dabei erachten wir es für notwendig, zu betonen, dass auch wir zutiefst empört sind über die Gewalt an Grenzen, die den Anstoß zu diesen kritischen Untersuchungen gibt. Wir sind dennoch überzeugt, dass eine politische und juristische Kritik um eine Kritik der politischen Ökonomie der Grenze erweitert werden muss, ein Aspekt, der heute oft vernachlässigt wird.

Wenn wir den Fokus der Analyse auf die politische Ökonomie der Grenze richten, müssen wir die unterschiedlichen Formen der Produktion des Raumes hervorheben, die mit den zwei wesentlichen, die heutige Zeit kennzeichnenden Gewalten verknüpft sind: Staat und Kapital. Während die Existenz des Staates auf linear gezogenen Grenzen beruht (die in der kolonialen und imperialen Herrschaft außerhalb Europas mannigfaltige und fragmentierte Züge annehmen), erscheint aus Sicht des Kapitals - um aus Marx' Werk Grundrisse zu zitieren (Marx 
1857-1858/1967, S. 311) - „jede Grenze [...] als zu überwindende Schranke“ und operiert damit letztendlich im konzeptuellen Rahmen des „Weltmarktes“ (ebd.). Indem wir uns der Mehrdeutigkeit des semantischen Feldes der Grenze bedienen, richten wir also die strittige Frage der Beziehung zwischen Staat und Kapital unter Berücksichtigung der Spannungen (und notwendigen Verknüpfungen) zwischen einer großen Bandbreite territorialer Grenzen und den von uns sogenannten expansiven Grenzen des Kapitals neu aus. Und wir zeigen auf, wie wichtig es ist, aus historischer Perspektive zu untersuchen, auf welche Art und Weise sich die politischen Landkarten zu den Landkarten der kapitalistischen Akkumulation verhalten. Indem wir das tun, diskutieren wir aus Sicht der Grenze so wichtige Fragen wie zum Beispiel Theorien des Imperialismus und der räumlichen Nomenklatur, die durch die Weltsystemtheorie verfälscht wurden (Zentrum, Semiperipherie, Peripherie) (vgl. Wallerstein 1974; Mezzadra/Neilson 2013).

Von zentraler Bedeutung für unsere Analyse ist unsere Skepsis gegenüber dem Konzept internationaler Arbeitsteilung, die im Nachgang des in den 1970er Jahren beginnenden Outsourcens der industriellen Produktion ihren Anfang nahm und seit den 1980er Jahren weithin als ,neue' internationale Arbeitsteilung verstanden wird. Sie sorgt auch weiterhin für kritische Debatten. Grenze als epistemologische Perspektive und als Methode zu verankern, erscheint uns in diesem Zusammenhang als ausgesprochen hilfreich. Unsere genealogische Untersuchung der internationalen Arbeitsteilung basiert auf der Betonung des Bordering-Prozesses, der in der zeitgenössischen Grenzforschung weit verbreitet ist - das bedeutet auf der Denaturalisierung der Grenze und auf einem kritischen Augenmerk gegenüber der Art und Weise, wie das Ziehen von Grenzen zur tatsächlichen Erschaffung der Welt beiträgt; wir bezeichnen dies als „fabrica mundi“ (Mezzadra/Neilson 2013, Kap. 2) und greifen damit den Titel des Atlas“ von Gerhard Mercator (1595) auf. Wir sind weit davon entfernt, die internationale Welt als unumstößliche Gegebenheit zu betrachten. Vielmehr versuchen wir, die Aufmerksamkeit auf die tumultartige und konfliktbehaftete Dynamik der Nationalisierung der Welt zu lenken, welche das Fundament für die Entstehung der nationalen und industriellen Schubkraft in der Geschichte des Kapitalismus im 19. und 20. Jahrhundert bildet. Die internationale Arbeitsteilung ist ein konzeptueller Rahmen, der diese Dynamik gleichermaßen widerspiegelt und befeuert, wie das Ziehen von Grenzen, mit denen nationale Märkte eingeschränkt werden und die eine spezifische Form der Struktur des Weltmarktes ermöglichen - in all seiner dramatischen Unausgewogenheit und seiner von Herrschaft und Abhängigkeit gezeichneten Geografie.

Unsere Analyse unterstreicht insbesondere die Bedeutung von Grenzen in Bezug auf den Aufbau sowie die Organisation und Regulierung des Arbeitsmarktes. Und sie lädt dazu ein, aus dieser Perspektive die sich verändernden Rekrutierungs- und Managementregime zu betrachten, denen die Arbeit Migrierender im Kapitalismus unterliegt. Indem wir die Vorrangstellung der Mobilität betonen, die unserem Ansatz zugrunde liegt, zeigen wir im letzten Teil des Beitrages auf, welche Bedeutung die Praktiken, Routinen und Konflikte der Migrierenden haben, mit denen diese gegen den sogenannten ,Fordismus‘ ankämpfen und gleichzeitig auch die damit verbundene Einwanderungspolitik in Frage stellen, die in Westeuropa seit 1955 sinnbildhaft durch das ,Gastarbeiter'-Projekt der westdeutschen Regierung verkörpert wird. Unser besonderes Interesse gilt dabei den Veränderungen, denen der Kapitalismus unterworfen ist und die sich in vielerlei Hinsicht seit den frühen 1970er Jahren vollziehen. Wir sind davon überzeugt, dass aufgrund dieser Veränderungen sowohl die expansiven Grenzregionen des Kapitals als auch die territorialen Grenzen strukturell aus den Fugen geraten sind. Auf den fol- 
genden Seiten werden wir uns auf die Prozesse der Vervielfältigung der Arbeit (multiplication of labor) konzentrieren, durch die die traditionellen Theorien der Arbeitsteilung zunehmend komplexer und umfangreicher geworden sind. Diese Prozesse sorgen für eine exponentielle Zunahme von Grenzen, die wir hier aus der Perspektive der damit verbundenen Diversifizierung der lebendigen Arbeit und der Migration analysieren. Darüber hinaus nehmen wir eine Neueinordnung solch wichtiger politischer Konzepte wie Klassen und Internationalismus aus der Perspektive der Grenze vor.

\section{Konzeptueller Rahmen}

Unser Interesse gilt den sich verändernden Grenz- und Migrationsregimen in einer Welt, in der Nationalgrenzen nicht mehr die einzigen oder notwendigerweise die wichtigsten Grenzen darstellen, um die Mobilität der Arbeitenden zu entflechten und zu beschränken. Der Nationalstaat ist immer noch eine wichtige politische Bezugsgröße, wenn es um Machtkonfigurationen und ihre Beziehungen zwischen Kapital und Arbeit geht. Wir sind jedoch davon überzeugt, dass aktuelle Machtdynamiken und -kämpfe nicht durch nationale Grenzen oder die internationalen Systeme, die sie vermeintlich etablieren, kontrolliert werden können. Dies ist ein wichtiger Ausgangspunkt für unsere Arbeit. Zwar betonen wir die strategische Bedeutung, die Grenzen in der heutigen Welt haben, aber wir beabsichtigen mitnichten, in den Chor einzustimmen, der in den vergangenen Jahren und von vielen unterschiedlichen Standpunkten aus die Wiederkehr des Nationalstaates auf der Weltbühne feierte und gleichzeitig die Debatten über die Globalisierung als bloße ideologische Verirrung verwarf. Im Gegenteil, eine unserer zentralen Thesen besagt, dass Grenzen zu einem entscheidenden Mittel der Verknüpfung geworden und weit davon entfernt sind, allein dazu zu dienen, globale Ströme zu unterbinden oder zu erschweren. Aus diesem Grund haben Grenzen nicht nur eine unaufhaltsame Ausbreitung erfahren, sie unterliegen auch komplexen Veränderungen, die mit dem einhergehen, was Saskia Sassen (2007, S. 214) als „the actual and heuristic disaggregation of ,the border“" bezeichnet hat. Die vielschichtigen (rechtlichen und kulturellen, sozialen und wirtschaftlichen) Komponenten sowohl des Konzeptes als auch der Institution der Grenze scheinen sich allmählich von der magnetischen Linie, die der geopolitischen Trennlinie zwischen Nationalstaaten entspricht, zu lösen. Um diesen Prozess erfassen zu können, nehmen wir eine kritische Distanz zu dem vorherrschenden Interesse an geopolitischen Grenzen ein, das viele kritische Forschungsansätze auszeichnet, und sprechen nicht nur von der ungebremsten Zunahme, sondern auch von einer Heterogenisierung von Grenzen.

Das traditionelle Bild der Grenze ist immer noch in Landkarten festgeschrieben, auf denen einzelne souveräne Gebiete durch Linien voneinander getrennt und mit unterschiedlichen Farben markiert sind. Dieses Bild ist geprägt durch die moderne Geschichte des Nationalstaates, deren Komplexität wir uns immer vor Augen halten müssen. Um nur ein Beispiel zu nennen: Die Migrationskontrolle ist erst in jüngerer Zeit zu einer bedeutenden Funktion der politischen Grenze geworden. Gleichzeitig führt die geschichtliche Aufarbeitung der Entwicklung linearer Grenzen mit dazu, dass wir uns zunehmend des Risikos bewusst sind, das es mit sich bringt, sich ein bestimmtes Bild von der Grenze anzueignen. Denn dieses Bild ist nicht hilfreich, um die augenfälligsten Veränderungen zu verstehen, denen wir uns heute stellen müssen. Heute markieren Grenzen nicht einfach nur geografische Randgebiete oder Grenzregionen. Sie stellen vielmehr komplexe soziale Institutionen dar, die von Spannungen zwischen den Praktiken der Grenzbewehrung und denen des Grenzübertritts gekennzeichnet sind. Diese Definition von 
Grenze, die auf einen Versuch von Pablo Vila (2000) zurückgeht, die Forschungsgeschichte der US-mexikanischen Grenzregion seit den späten 1980er Jahren aufzuarbeiten, zeigt die Spannungen und Konflikte auf, die für jede Grenze kennzeichnend sind.

Wir sind davon überzeugt, dass dieses konstituierende Element heute mit besonderer Intensität zu Tage tritt, sowohl entlang spezifischer geopolitischer Grenzen als auch entlang vieler anderer Trennlinien, die mitten durch Städte, Regionen und Kontinente verlaufen. Einerseits werden Grenzen zu besonders fein abgestimmten Instrumenten, um den globalen Verkehr von Menschen, Geld und Waren zu lenken, zu bewerten und zu verwalten. Andererseits stellen sie Räume dar, in denen die Transformationen, denen die Staatsgewalt unterworfen ist, und die ambivalenten Verflechtungen von Politik und Gewalt niemals wirklich außer Sicht geraten. Bei der Beobachtung dieser zwei Tendenzen geht es nicht nur darum, die banale, aber notwendige Feststellung zu treffen, dass Grenzen immer zwei Seiten haben oder dass ihnen sowohl eine verbindende als auch eine separierende Funktion zukommt. Grenzen übernehmen auch eine wesentliche Funktion der Produktion von Räumen und Zeiten für den global agierenden Kapitalismus. Darüber hinaus prägen sie die Konflikte, die innerhalb dieser und gegen diese Räume und Zeiten entstehen - Konflikte, die häufig auf problematische, aber durchaus vielfältige und determinierte Art und Weise auf die Abschaffung der Grenze selbst abzielen. In dieser Hinsicht sind Grenzen in den letzten Jahren zu einem wichtigen Thema in der Wissenschaft sowie im politischen und künstlerischen Alltag geworden. Sie sind Orte, an denen die Turbulenzen und die konfliktbehaftete Intensität der globalen kapitalistischen Dynamik besonders deutlich zu Tage treten. Als solche liefern sie die strategische Basis für die Analyse und Verachtung der gegenwärtig existierenden Globalisierung.

\subsection{Was ist eine Grenze?}

In einem richtungsweisenden Essay mit dem Titel What Is a Border? schreibt Étienne Balibar (2002, S. 76) über die Polysemie und Heterogenität von Grenzen und bemerkt: „[their] multiplicity, their hypothetical nature [does] not make them any less real“. Es gibt nicht nur unterschiedliche Arten von Grenzen, die von Individuen, die unterschiedlichen sozialen Gruppen angehören, auf unterschiedliche Art und Weise wahrgenommen werden. Darüber hinaus leisten Grenzen zugleich „several functions of demarcation and territorialisation - between distinct social exchanges or flows, between distinct rights, and so forth“ (ebd., S. 79). Mehr noch, Grenzen sind immer überbestimmt (overdetermined), das heißt:

„no political border is ever the mere boundary between two states, but is always [...] sanctioned, reduplicated and relativized by other geopolitical divisions. [...] Without the world-configuring function they perform there would be no borders - or no lasting borders" (ebd.).

Seine Begründung erinnert an einen Text, der in einem ganz anderen theoretischen Kontext von Carl Schmitt (1950) in Der Nomos der Erde entwickelt wurde und in dem die Behauptung aufgestellt wird, dass Grenzziehungen innerhalb des modernen Europas Hand in Hand mit rechtlichen Arrangements gingen, die darauf ausgerichtet waren, einen bereits existierenden globalen Raum zu organisieren. Diese Arrangements, die unterschiedliche Arten „globaler Linien“ (Schmitt) und geografischer Einteilungen umfassten, erwiesen sich als Blaupause für die koloniale Aufteilung der Welt und die Steuerung der Beziehungen zwischen Europa und 
seinen umgebenden Ländern. Kurz, die Verflechtung dieser globalen Linien der imperialistischen Expansion mit der linearen Grenzziehung zwischen europäischen und westlichen Staaten hat über mehrere Jahrhunderte hinweg das vorherrschende Motiv der globalen, von Kapital und Staat organisierten Geografie konstituiert. Diese Geschichte verlief ganz offensichtlich weder friedlich noch linear. Auch wenn die politische Landkarte der Welt und die globale Kartographie des Kapitalismus niemals völlig deckungsgleich waren, so konnten sie einst doch leicht voneinander abgelesen werden. In der Welt des Kalten Krieges war die Überdeutlichkeit dieser Karten zunehmend schwerer zu lesen. Eine Kombination aus unterschiedlichsten Prozessen der „Denationalisierung“ (Sassen 2006) hat sowohl den Staat als auch das Kapital mit unterschiedlicher Intensität und in unterschiedlichem Ausmaß erfasst. Insbesondere der nationale Nennwert des Kapitals als signifikanter Index für eine Analyse des aktuellen Kapitalismus verlor immer weiter an Bedeutung. Wir gehen dieses Problem an, indem wir das Konzept der Randzonen des Kapitals (frontiers of capital) herausarbeiten und die Beziehungen untersuchen, die zwischen der kontinuierlichen Expansion dieser Randzonen seit dem Entstehen des modernen Kapitalismus und den territorialen Grenzen bestehen. Obwohl diese Beziehungen schon immer durch eine ihnen innewohnende Spannung geprägt waren, hat die Entwicklung des Kapitalismus als Weltsystem zu unterschiedlichen Spielarten der Verknüpfung zwischen den Grenzlinien, die durch wirtschaftliche Prozesse hervorgerufen wurden, und den Staatsgrenzen geführt. Einer unserer zentralen Standpunkte lautet, dass das gegenwärtige Kapital, charakterisiert durch Finanzialisierungsprozesse und durch die Kombination heterogener Arbeits- und Akkumulationsregime, die Expansion seines Geltungsbereiches auf Basis einer viel komplexeren Zusammenballung von Macht und Gesetz verhandelt, die Nationalstaaten gleichermaßen einschließt wie transzendiert. Wenn wir einen Blick auf die Expansion des Geltungsbereichs des Kapitals werfen und uns die Ausweitung der politischen und rechtlichen Grenzen vor Augen halten, dann werden wir mit einem geographischen Umbruch und einem andauernden Prozess des rescaling konfrontiert. Diesem Prozess steht ein zutiefst heterogener globaler Raum gegenüber, wobei die Grenze eine besonders effektive Perspektive darstellt, aus der seine Entstehung untersucht werden kann.

Grenzen üben heute noch immer eine „weltkonfigurierende Funktion“ (Balibar) aus, aber sie sind häufig sich verändernden und unvorhersagbaren Mustern der Mobilität unterworfen, überschneiden sich, tauchen auf und verschwinden wieder oder materialisieren sich in Form bedrohlicher Mauern, die auseinanderbrechen und politische, einst formal vereinte Räume neu ordnen. Sie durchziehen das Leben von Millionen von Männern und Frauen, die in Bewegung sind oder, wenn sie sesshaft bleiben, durch Grenzen voneinander getrennt werden. Gleichzeitig überlagern Grenzen andere Formen von Begrenzungen und Technologien der Teilung. Diese Prozesse sind nicht weniger überbestimmt als die der modernen Weltordnung, aber die Art und Weise, wie sie den Globus konfigurieren, hat sich dramatisch verändert. Statt eine stabile Karte der Welt zu zeichnen, zielt der Prozess der Ausweitung und Transformation von Grenzen, die wir analysieren, darauf ab, die kreative Zerstörung und die konstante Neuzusammensetzung von Räumen und Zeiten zu managen, die das Herzstück der gegenwärtigen kapitalistischen Globalisierung bilden.

Wir sind geneigt, Grenze als physische und metaphorische Mauer zu betrachten, so wie es uns durch das Bild der Festung Europa eingeflüstert wird. Das scheint nach den Ereignissen des 11. September 2001 umso mehr der Fall zu sein, als Grenzen zu wichtigen Orten wurden, an denen sich das ,Sicherheits'-Paradigma innerhalb der politischen Rhetorik sowie der eigentlich 
auf Kontrolle zielenden Politik manifestierte (siehe zum Beispiel Longo 2017). Wir sind uns all dessen schmerzlich bewusst. Dennoch sind wir davon überzeugt, dass das Bild der Grenze als Mauer oder als Mittel, das in erster Linie und hauptsächlich dazu dient, auszuschließen, so intensiv es auch in jüngeren kritischen Studien behandelt wurde, am Ende in die Irre führt. Eine einzelne Funktion der Grenze zu isolieren, erlaubt uns nicht, die Flexibilität dieser Institution zu erfassen. Und ebenso wenig erleichtert es uns, die Durchlässigkeit von Praktiken und Techniken der Grenzkontrolle innerhalb territorial abgesteckter Räume von Nationalitäten und den mit ihnen assoziierten Arbeitsmärkten zu verstehen. Wir behaupten, dass Grenzen sowohl Mittel der Inklusion sind, die Menschen und verschiedene Formen der Zirkulation auf eine Art und Weise selektieren und filtern, in der nicht weniger Gewalt angewendet wird als durch exkludierende Maßnahmen. Unsere Diskussion nähert sich der Inklusion, die in den meisten Fällen als harmloses soziales Gut angesehen wird, deshalb kritisch. Indem wir zeigen, inwiefern Grenzen vielfältige Kontrollpunkte entlang der wesentlichen Linien und Topographien von Wohlstand und Macht etablieren, betrachten wir Inklusion als Fortsetzung der Exklusion und nicht als deren Gegenteil. Mit anderen Worten: Wir konzentrieren uns auf die hierarchisierenden und schichtenden Merkmale von Grenzen, indem wir ihren Bezug zu Kapital und politischer Macht untersuchen, egal ob sie mit den territorialen Grenzen des Staates zufällig zusammen fallen oder innerhalb oder außerhalb dieser Grenzen existieren. Um den pervasiven Charakter des Wirkens von Grenzen zu analysieren - um nicht zu sagen die ausgeprägte Gewalt, die mit ihnen verbunden ist -, benötigen wir eine komplexere und dynamischere konzeptuelle Sprache als die, die das Bild von Mauern und Exklusion aufrechterhält. Grenze als Methode führt eine Bandbreite von Konzepten ein, mit denen der Versuch unternommen wird, die schleichenden Veränderungen zu erfassen, denen Arbeit, Raum, Zeit, Recht, Macht und Bürgerrechte ausgesetzt sind und die die Ausbreitung von Grenzen in der heutigen Welt begleiten. Darunter fallen die Vervielfältigung der Arbeit, eine differenzierende Inklusion, zeitliche Grenzen, der Machtapparat der Verwaltung und Konflikte an Grenzen. Zusammengenommen stellen diese Konzepte ein Netz dar, innerhalb dessen die tiefen Veränderungen der sozialen, wirtschaftlichen, rechtlichen und politischen Beziehungen auf unserem Planeten erkennbar werden. Sie verweisen auf einen radikal äquivoken Charakter von Grenzen und auf ihre zunehmende Unfähigkeit, eine beständige Trennlinie zwischen dem Innen und Außen eines Territorialstaates zu ziehen.

Die Einzigartigkeit unseres Ansatzes besteht in dem Versuch, die Grenze von der Mauer zu trennen, indem wir zeigen, auf welche Weise die regulativen Funktionen und die symbolische Macht der Grenze die Schwelle zwischen Staatsgewalt und eher flexiblen Formen der globalen Verwaltung auf die Probe stellen und somit wie ein Prisma wirken, durch das wir die Transformationen des Kapitals ebenso nachvollziehen können wie die Kämpfe, die innerhalb und gegen sie stattfinden. Die Aufmerksamkeit gegenüber der historischen und geographischen Bedeutung einzelner Grenzen würdigt keineswegs einen Ansatz herab, der bestimmte Aspekte einer Situation isoliert, und bringt sie in Einklang mit dem, was in sehr unterschiedlichen Raum- und Zeitzonen geschieht.

Unser Ziel ist es, eine Reihe von Problemen, Prozessen und Konzepten in den Fokus zu rücken, die es uns erlauben, einen neuen theoretischen Ansatz der Grenze herauszuarbeiten. Indem wir das tun, gehen wir auf Distanz zu einer Rhetorik, die sich auf das Bild einer Mauer oder auf das Thema der Sicherheit beschränkt. Wir verabschieden uns auch von dem klassischen Paradigma der Grenzforschung (vgl. Kolossov 2005; Newman 2006), die dazu neigt, sich auf 
den Vergleich einzelner Fallstudien zu beschränken, und auf der Annahme beruht, es gebe eindeutige und spezifische Differenzierungsmerkmale zwischen den verschiedenen Situationen und Kontexten, die erforscht werden.

Wenn wir über Grenzkonflikte schreiben, dann ist das für uns eine Möglichkeit, den Fokus auf die Produktion politischer Subjektivität zu richten. Wir sind nicht nur an Bewegungen interessiert, die Grenzen und ihre diskriminierenden Eigenschaften offen ablehnen, wie diejenigen, in denen Migrierende ohne Papiere zu Protagonistinnen und Protagonisten werden (vgl. SuárezNaval et al. 2007). Wir möchten die Erwähnung der Konflikte an Grenzen auch nutzen, um auf die alltäglichen Praktiken hinzuweisen, durch die Migrierende sich regelmäßig mit den alles durchdringenden Effekten der Grenze arrangieren, indem sie sich ihnen entziehen oder ihnen durch den Aufbau von Netzwerken und transnationalen sozialen Räumen begegnen (vgl. Rodríguez 1996). Zudem wollen wir aufzeigen, inwiefern Konflikte an Grenzen - die immer durch spezifische individuelle Positionen und Personen geprägt sind - das Feld der politischen Subjektivität generell durchdringen, deren intrinsische Grenzen auf die Probe stellen und ihre interne Einteilung neu organisieren. Auf diese Weise eröffnen Auseinandersetzungen an Grenzen einen völlig neuen Kontinent politischer Möglichkeiten, einen Raum, in dem neuartige politische Subjekte, die sich weder der Logik der Staatsbürgerschaft noch den etablierten Methoden radikaler politischer Organisation und politischen Handelns unterwerfen, ihre Kreise ziehen und ihre Macht vergrößern können. Die Erforschung dieses Kontinents setzt mit den materialen Bedingungen ein, die die Spannung hervorbringen, deren sichtbares Zeichen diese Auseinandersetzungen sind. Dieser Ansatz scheint uns vielversprechender - und in politischer Hinsicht drängender - zu sein, als den simplen Vorwurf zu formulieren, Grenzen wirkten ausschließend, oder aber den Wunsch nach einer Welt ,ohne Grenzen' auszusprechen. Mehr als einmal haben wir an Balibars Erwähnung der Polysemie der Grenze erinnert, ein Konzept, das mit der Vielschichtigkeit von Begriffen korrespondiert, die in vielen Sprachen auf das semantische Feld der Grenze verweisen (denken Sie nur an die englischen Begriffe boundary und frontier). Es ist kein Zufall, dass der metaphorische Gebrauch dieser Begriffe heute weitverbreitet ist (vgl. Newman/Paasi 1998). Das fällt nicht nur in der Alltagssprache auf (z.B. Grenzen wissenschaftlicher Forschung), sondern auch in der Fachsprache der Sozialwissenschaften, wo Phrasen wie boundary work oder boundary object Eingang in den allgemeinen Sprachgebrauch gefunden haben (vgl. Lamont/Molnár 2002). Neben ihrer geographischen, politischen und rechtlichen Dimension hat das Konzept der Grenze eine wichtige symbolische Dimension, die aufgrund der Zunahme der Spannungen, die die üblicherweise moderne Konfiguration der Grenze als separierende Linie zwischen souveränen Staatsterritorien verdrängt haben, ins Blickfeld gerückt ist (vgl. Zanini 1997; Cella 2006). Sowohl die Soziologie, anknüpfend an die Arbeit von Georg Simmel (1908/2009), als auch die Anthropologie, ausgehend von einem richtungsweisenden Essay von Fredrik Barth (1979), haben fundamentale Beiträge geleistet, um diese symbolische Dimension der Grenze zu verstehen, darunter auch ihre Rolle im Hinblick auf die Differenzierung sozialer Formen und die Organisation kultureller Unterschiede.

Die Unterscheidung zwischen den Begriffen border und frontier ist unzweifelhaft wichtig (vgl. Prescott 1987). Erstere wird typischerweise als Linie angesehen, während letztere als offener und sich ausdehnender Raum betrachtet wird. In vielen zeitgenössischen Kontexten scheint diese Unterscheidung jedoch zu verwischen. Die Grenzen des gegenwärtigen europäischen Raumes beispielsweise zeichnen sich durch Aspekte der Unbestimmtheit aus, die historisch die Grenzregionen (frontiers) charakterisierten. Sie dehnen sich in deren umgebende Gebiete 
aus und schaffen an diese variable Geometrie angepasste Räume, die mit vielfältigen geographischen Größen verknüpft sind (vgl. Cuttitta 2007). Grenze als Methode handelt von solchen Momenten schwieriger konzeptueller Überschneidung und Unübersichtlichkeit durch die punktuelle Analyse konkreter Grenzlandschaften (borderscapes). In jedem Fall ist die Grenze für uns mehr als ein Forschungsobjekt, das verschiedenen methodologischen Ansätzen zugeordnet werden kann und mehr als ein semantisches Feld, dessen multiple Dimensionen erforscht werden müssen. Insofern, als sie in erster Linie dazu dient, Unterteilungen vorzunehmen und Verbindungen zu etablieren, ist die Grenze ein epistemologisches Mittel, das immer dann in Funktion tritt, wenn eine Unterscheidung zwischen Subjekt und Objekt hergeleitet wird. Um es noch einmal zu betonen: Balibar beschreibt diesen Aspekt von Grenze höchst präzise, indem er die Schwierigkeit erwähnt, die der Definition des Konzeptes selbst innewohnt:

"The idea of a simple definition of what constitutes a border is, by definition, absurd: to mark out a border is precisely, to define a territory, to delimit it, and so to register the identity of that territory, or confer one upon it. Conversely, however, to define or identify in general is nothing other than to trace a border, to assign boundaries or borders (in Greek, horos; in Latin, finis or terminus; in German, Grenze; in French, borne). The theorist who attempts to define what a border is is in danger of going round in circles, as the very representation of the border is the precondition for any definition " (Balibar 2002, S. 76, Herv. i. O.).

Grenzen sind also von entscheidender Bedeutung für kognitive Prozesse, weil sie es erlauben, die Etablierung sowohl von Taxonomien als auch von konzeptuellen Hierarchien vorzunehmen, die die Bewegung der Gedanken strukturieren. Darüber hinaus ermöglichen sie eine wissenschaftliche Arbeitsteilung, die mit der Einteilung von Wissen in unterschiedliche Disziplinen assoziiert wird.

\subsection{Grenze als Methode}

Einerseits beziehen wir uns auf einen Prozess der Wissensproduktion, der die Spannung zwischen empirischer Forschungsarbeit und der Erfindung von Konzepten, die dieser zugrunde liegen, aufrechterhält. Andererseits bedeutet der Ansatz der Grenze als Methode, dass wir uns, um auf eine phänomenologische Kategorie zurückzugreifen, der an einzelne Disziplinen geknüpften Praktiken entledigen, die die Objekte des Wissens als bereits konstituiert begreifen, und stattdessen die Prozesse untersuchen, durch die diese Objekte konstituiert werden. Indem wir das konstituierende Moment der Grenze retten und reaktivieren, versuchen wir uns den Teufelskreis, den Balibar identifiziert, nutzbar zu machen. Ebenso wie wir die Vision von Grenze als neutraler Linie in Frage stellen wollen, bezweifeln wir auch die Annahme, dass eine Methode eine Auswahl an vorgegebenen, neutralen Techniken ist, die auf unterschiedliche Objekte angewandt werden können, ohne grundsätzlich die Art und Weise zu verändern, wie sie konstruiert sind und verstanden werden. Das Entscheidende am Konzept der Grenze als Methode ist mehr als nur die „Performativität der Methode“ (Law 2004, S. 149) und auch mehr als die bestechende Idee der analytic borderlands (Sassen 2006, S. 379-386). Das heißt, auch wenn wir akzeptieren, dass Methoden üblicherweise (häufig auf widersprüchliche und unerwartete Weise) die Welten produzieren, die sie eigentlich beschreiben wollen, ist doch die Frage nach der Grenze als Methode etwas mehr als nur ein methodologisches Phänomen. Es 
ist vor allem eine Frage der Politik, nach der Vielzahl sozialer Welten und Subjektivitäten, die an der Grenze produziert werden, sowie nach den Wegen, auf denen Gedanken und Wissen in diese Produktionsprozesse einfließen können. Um es anders auszudrücken: Wir können festhalten, dass Methode für uns in vielerlei Hinsicht sowohl das Handeln in der Welt als auch das Wissen über sie umfasst. Genauer gesagt geht es um die Beziehung von Handeln und Wissen in einer Situation, in der viele unterschiedliche Wissensregime und -praktiken in Konflikt miteinander geraten. Grenze als Methode bedeutet auch, die unterschiedlichen Arten des Wissens auszuhandeln, die an der Grenze zum Tragen kommen, und zielt, indem das geschieht, darauf ab, Licht auf die Subjektivitäten zu werfen, die durch solche Konflikte entstehen.

Aus all diesen Gründen ist Grenze für uns weniger ein Forschungsobjekt als eine epistemologische Perspektive, die uns eine exakte und kritische Analyse zum einen der Art und Weise erlaubt, wie Beziehungen der Herrschaft, Besitzlosigkeit und Ausbeutung gegenwärtig neu definiert werden, und zum anderen der Auseinandersetzungen, die rund um diese sich verändernden Beziehungen Form annehmen. Die Grenze kann genau insofern eine Methode sein, als sie als Ort wahrgenommen wird, an dem Konflikte ausgetragen werden. Wie wir bereits hervorgehoben haben, sind unsere Forschungsarbeit und unsere theoretischen Arbeiten vor allem durch die Intensität der Konflikte initiiert, die wir an Grenzen überall in der Welt finden können. Sobald wir die vielschichtigen Praktiken untersuchen, mit denen Migrierende jeden Tag Grenzen in Frage stellen, wird offensichtlich, dass Konflikte an Grenzen häufig tatsächlich eine Sache auf Leben und Tod sind. Auch wenn wir ein weiter angelegtes Konzept von Auseinandersetzungen an Grenzen ausarbeiten, das mit der ausufernden Zunahme und Heterogenität von Grenzen in der heutigen Zeit korrespondiert, vergessen wir diese Beschaffenheit der Grenze nicht. Den Fokus auf Konflikte an der Grenze zu richten, stellt auch die Exaktheit der Grenze als Methode sicher. Es erlaubt uns nicht nur, eine Auswahl der relevanten empirischen Settings für unsere Untersuchungen zu treffen, sondern auch, die gesamte Konstruktion der ,Objekte', die erforscht werden sollen, zu erfassen. In jedem Fall liegen unserer Analyse spezifische Landschaften, Praktiken und Grenztechnologien zugrunde. Die Methode, die wir verfolgen, entsteht aus einer kontinuierlichen Auseinandersetzung mit den Merkmalen der Spannungen und Konflikte, die die Grenze als Institution und als Geflecht sozialer Beziehungen konstituieren. Selbst wenn wir uns mit augenscheinlich abstrakten Themen wie beispielsweise dem Übersetzen beschäftigen, versuchen wir, diesen Merkmalen Beachtung zu schenken.

Wir bemühen uns, eine Perspektive auf die Grenze, die durch unser Interesse an der menschlichen Arbeitskraft gekennzeichnet ist, mit unserem Interesse an Grenzkonflikten und der Produktion von Subjektivität in Einklang zu bringen. Unsere Analyse konzentriert sich entsprechend auf die dichten und konfliktbehafteten Wege, auf denen Grenzen das Leben und die Erfahrungen von Subjekten formen, die aufgrund des Funktionierens der Grenze selbst als Träger der Arbeitskraft konfiguriert sind. Die Produktion der Subjektivität dieser Subjekte konstituiert einen wesentlichen Moment innerhalb des allgemeineren Prozesses, in dem Arbeitskraft als Ware produziert wird. Sobald sie aus dieser Perspektive betrachtet werden, müssen sowohl die Techniken der Macht, die Grenzen beinhalten, als auch die sozialen Praktiken und Konflikte, die sich daraus entwickeln, im Hinblick auf die multiplen und instabilen Konfigurationen von Geschlecht und Ethnie, Produktion und Reproduktion analysiert werden, durch die sie selbst in hohem Maße an der Grenze beeinflusst werden. Wenn wir feststellen, dass die Grenze 
eine entscheidende Rolle in der Kommodifizierung von Arbeitskraft spielt, dann müssen wir auch diskutieren, dass die Art und Weise, wie Migrationsbewegungen durch Grenzregime kontrolliert, gefiltert und unterbunden werden, allgemeinere Effekte auf die politische und rechtliche Beschaffenheit von Arbeitsmärkten und damit auch auf die Erfahrung lebendiger Arbeit haben. Wir zeigen, dass die Konflikte, die sich um diese Erfahrungen entwickeln, ob nun zentral organisiert oder autonom, immer eine Konfrontation mit der Frage der Grenze implizieren. Außerdem zeigen wir in diesem Kontext auf, dass das Übersetzen eine wichtige Rolle in der Erfindung neuer Formen der Organisation und neuer sozialer Institutionen spielen kann.

\section{Die Vervielfältigung der Arbeit}

Man könnte sagen, dass die Vervielfältigung immer ein wichtiger Punkt in Debatten über (und praktischen Übersetzungen von) Arbeitsteilung war. Teilung hat immer die Vervielfältigung (der Produktivität, der Menge, des Wohlstands usw.) zum Ziel gehabt. Adam Smith (1776/2009, S. 18) schreibt zum Beispiel:

„Ebendie große durch die Arbeitsteilung bewirkte Vervielfältigung der Produkte in allen verschiedenen Künsten ist es, die in einer wohlregierten Gesellschaft jene allgemeine Wohlhabenheit hervorbringt, die sich selbst bis zu den untersten Klassen des Volkes erstreckt.“

Hinter dieser Aussage können wir das Problem der Beziehung (und der möglichen Spannungen) zwischen sozialer Kooperation und der Spezialisierung sozialer Funktionen erkennen, die ursprünglich von David Hume formuliert worden sind. „Durch die Vereinigung der Kräfte“, schreibt Hume in seinem Traktat über die menschliche Natur (1739/1973, S. 229), „wird unsere Leistungsfähigkeit vermehrt; durch Teilung der Arbeit wächst unsere Geschicklichkeit, und gegenseitiger Beistand macht uns weniger abhängig von Glück und Zufall“.

In seiner Analyse Maschinerie und große Industrie spricht Marx (1867/1986, S. 511) von einem „absoluten Widerspruch“ zwischen den revolutionären Tendenzen der Industrie einerseits, die kontinuierlich „die Funktionen der Arbeiter und die gesellschaftlichen Kombinationen des Arbeitsprozesses um[wälzen] “, und den Bedürfnissen des Kapitalisten andererseits, “die alte Teilung der Arbeit mit ihren knöchernen Partikularitäten“ zu reproduzieren. Er fügt hinzu: „Die Natur der großen Industrie bedingt daher Wechsel der Arbeit, Fluß der Funktion, allseitige Beweglichkeit des Arbeiters“ (ebd.), während das Kapital gezwungen ist, diese Prozesse kontinuierlich zu begrenzen, einzuschränken und zu unterbinden.

Die Krise des Taylorismus und des Fordismus, die in den 1980er Jahren allerorten diskutiert wurde, kann anhand dieser Zeilen nachvollzogen werden, auch wenn man anmerken muss, dass es in erster Linie die Forderungen nach Variation, Fluidität und Beweglichkeit und die entsprechende Umsetzung durch die Arbeiter waren, die die Krise beschleunigten (vgl. Boltanski/Chiapello 2005). Die Diskussion vor allem innerhalb der Unternehmen und in der Managementliteratur wurde von der Notwendigkeit beherrscht, den technischen Stillstand in der Organisation der Arbeit hinter sich zu lassen. ,Total Quality', ,das Japanische Modell und ,Toyotismus' waren die Slogans dieser Zeit und Das Ende der Arbeitsteilung?, der Titel eines gefeierten und einflussreichen Buches der deutschen Soziologen Horst Kern und Michael Schumann (1984). Uns geht es nicht in erster Linie darum, das ideologische Moment in diesen Diskursen und Strategien zu kritisieren: Es wäre leicht zu zeigen, wie schnell sich neue Formen 
des Stillstands in Total-Quality-Produktionsstätten und anderen Arbeitsumgebungen reproduzieren. Wir konzentrieren uns vielmehr darauf, wie das Kapital den von Marx konstatierten ,immanenten Widerspruch“ nach der Krise in den 1970er Jahren tatsächlich eingestand und managte. Es ist genau diese Perspektive, aus der uns das Konzept der Vervielfältigung der Arbeit als nützlich erscheint. Nachdem die Finanzialisierung neue Möglichkeiten bot, das Kapital angesichts der Beschränkungen, die durch die Arbeitenden innerhalb der Fabrikmauern gegeben waren, neu zu bewerten, riss das Kapital selbst diese Mauern nieder und sorgte für die Auslagerung der Arbeit nicht nur in geographischer Hinsicht, sondern auch über die gesamte Gesellschaft hinweg (vgl. Marrazzi 2010).

Arbeit wurde mit Hilfe dieser Prozesse in mindestens dreierlei Hinsicht vervielfältigt: Sie wurde erstens intensiviert, insofern als ihre Tendenz, das gesamte Leben der arbeitenden Subjekte zu kolonisieren, noch deutlicher zum Ausdruck kam als zuvor. Zweitens wurde sie intern diversifiziert, wie es ein Prozess nahelegt, den schon Marx in seiner Analyse des relativen Mehrwertes in den Grundrissen identifizierte und der das Kapital dauerhaft jenseits der Arbeitsteilung verortet, hin zur „Entwicklung von einem stets sich erweiternden und umfassenden System von Arbeitsarten, Produktionsarten, denen ein stets erweitertes und reichres [sic!] System von Bedürfnissen entspricht" (Marx 1857-1858/1967, S. 313). Drittens wurde sie in Bezug auf ihre rechtlichen und sozialen Systeme heterogenisiert.

Wenn wir der Marx'schen Analyse folgen, können wir die Einheit der zwei Dimensionen der kapitalistischen Transformation, die wir oben identifiziert haben (Finanzen und Arbeit) wiederum in ihrer vollen Bandbreite auf der Ebene des Weltmarktes erfassen: Wie wir in einer Passage seiner Theorien über den Mehrwert (Marx 1905-1910/1974, S. 250) gelesen haben, wird Geld nur dann zu „Weltgeld“, wenn sich ein Weltmarkt entwickelt, auf dessen Ebene wiederum „abstrakte Arbeit“ zu „gesellschaftlicher Arbeit“ wird. Der Weltmarkt ist mit anderen Worten der Ort der Repräsentation und der kontinuierlichen Reproduktion der kapitalistischen „Axiomatik“ (Deleuze/Guattari 1983), die letzte Garantie ihrer Herrschaft über die „den Weltmarkt umfassenden Totalität verschiedener Arbeitsweisen“ (Marx 19051910/1974, S. 250).

Um zu verstehen, wie Arbeit im Laufe der allgemeinen Krise der 1970er Jahre intensiviert wurde, ist es hilfreich, unterschiedliche zeitgenössische Arbeitsregime denen gegenüberzustellen, die Marx identifiziert hat. In Kapitel 15 des ersten Bandes seines Werkes Das Kapital (1867/1986) unterscheidet Marx drei wesentliche Faktoren, die die Abschöpfung des Mehrwertes beeinflussen: die Länge des Arbeitstages, die Produktivkraft und die Intensität der Arbeit. Auch wenn es möglich ist, dass alle drei Faktoren gleichzeitig variieren, gibt es eine Grenze, wie weit der Arbeitstag ausgedehnt werden kann, während die Intensität der Arbeit zunimmt. Diese Grenze ist durch die bloße Beschaffenheit des Körpers des Arbeiters vorgegeben, des lebendigen Faktors, der die abstrakte Menge der Arbeitskraft enthält. Es ist eine Begrenzung durch das, was ein Körper aushalten kann, bevor er zusammenbricht oder Ineffizienzen verursacht, die aus Erschöpfung, Krankheit oder der Unfähigkeit der Arbeit, sich auf täglicher Basis zu reproduzieren, resultiert. Diese Grenze, gegen die das Kapital ständig arbeitet, führt zu bestimmten Arrangements hinsichtlich technischer Aspekte der Arbeitsteilung, der Einrichtung von Schichtarbeit beispielsweise. Marx drückt es folgendermaßen aus:

„Indes begreift man, dass bei einer Arbeit, wo es sich nicht um vorübergehende Paroxysmen handelt, [...] ein Knotenpunkt eintreten muß, wo Ausdehnung des Arbeitstags und Intensität der Arbeit einander ausschließen, so daß die Verlängerung des Arbeitstags nur 
mit schwächerem Intensitätsgrad der Arbeit und umgekehrt ein erhöhter Intensivitätsgrad nur mit Verkürzung des Arbeitstags verträglich bleibt“ (Marx 1867/1986, S. 367).

Eine Möglichkeit, um die Intensivierung der Arbeit in einer Zeit, in der die Finanzialisierung des Kapitals neue Kanäle der Bewertung eröffnet hat, zu charakterisieren, besteht darin, festzustellen, dass diese Einschränkung und ihre gegenseitige Abhängigkeit, die sie zwischen der Intensität und der Extensität der Arbeit hervorbringt, noch nicht ausgewogen ist. Damit soll keineswegs geleugnet werden, dass auch weiterhin zahlreiche Fabriken und Produktionsstätten existieren - vom sweat shop bis zum Großraumbüro -, in denen eine Verschärfung dieser umgekehrten Beziehung zwischen der extensiven und intensiven Größe der Arbeit Körper und Leben der Arbeitenden unter anhaltenden Stress setzt. Aber diese Verschärfung, die die technische Koordinierung der Produktion entlang der globalen Wertschöpfungsketten begleitet hat, geht mit neuen Anforderungen an eine flexiblere und sozialere Beschaffenheit der Arbeit einher. Was wir an früherer Stelle als das Niederreißen der Fabrikmauern durch das Kapital beschrieben haben, schließt auch die Loslösung der Arbeit vom Maß der sozial erforderlichen Arbeitszeit ein. Kern ist hier weniger die Verlängerung des Arbeitstages als vielmehr die Tendenz der Arbeit, einen größeren Anteil des Lebens in Anspruch zu nehmen. Ob es das Vordringen der Arbeit in die heimische Sphäre oder der immer höhere Einsatz individueller Kommunikations- und Sozialfähigkeit zu Arbeitszwecken ist, die Tendenz der Arbeit, einen größeren Teil des Lebens zu kolonisieren, ist ein Faktor, der von vielen Kritikern und Kommentatoren beobachtet wird (vgl. z.B. Hochschild 1983; Virno 2003; Hardt/Negri 2004; Fumagalli 2007; Weeks 2007).

Diese Entwicklungen bringen nicht unbedingt eine Verringerung der Intensität der Arbeit mit sich. Die Beziehung der umgekehrten Proportionalität zwischen der Extensität und Intensität der Arbeit, wie sie von Marx beschrieben wurde, ist elastischer und verhandelbarer geworden. Es geht um die Produktion des absoluten und des relativen Mehrwertes, um die Aufteilung von bezahlter und unbezahlter Arbeit sowie um die zunehmende Verflechtung von produktiver und reproduktiver Arbeit. Das Fabrikenregime konnte für eine Ausgewogenheit der Belastungen durch die extensive und intensive Arbeit sorgen, bis exakt der Punkt erreicht war, an dem der Körper des Arbeiters zugrunde ging. In der fordistischen Ära entwickelten sich eine Reihe sozialer Institutionen, die zum Ziel hatten, die körperliche Unversehrtheit der Arbeitnehmerschaft zu gewährleisten. Zentraler Bestandteil dieses Arrangements war die Arbeitsteilung nach Geschlechtern zwischen Haushalt und Arbeitsplatz, wobei Ersterer der unbezahlten reproduktiven Arbeit diente und als Domäne der Frau galt, während Letzterer mit seiner bezahlten Arbeit dem Mann vorbehalten blieb. Mit dem Einsetzen der Finanzialisierung wurde der Haushalt selbst zum Ort kapitalistischer Berechnungen. Dick Bryan, Randy Martin und Mike Rafferty (2009, S. 462) schreiben, der Haushalt werde zunehmend wahrgenommen „[...] as a set of financial exposures to be self-managed“. Krankenversicherung, Bildungsausgaben, Hypotheken und Rentenversicherung sind nur einige der finanziellen Angelegenheiten, für die Haushalte die Verantwortung übernehmen. Letzten Endes hat die Reproduktion der Arbeitskraft die Tendenz, sich auf Kredite statt auf den Konsum von Gebrauchsgütern zu stützen und generiert somit Mehrwert auf Raten (in Form einer Rückzahlung), also indem Zinsen gezahlt werden. Wie in der durch Hypotheken ausgelösten Krise in den Jahren 2007/2008 sichtbar wurde, kann die Unfähigkeit der Arbeit, Kreditverpflichtungen zu begleichen, einen dramatischen Effekt auf die finanzielle Volatilität haben (vgl. Mezzadra/Neilson 2019, Kap. 4). 
Eine Folge dieser Prozesse der Finanzialisierung, die zuvor starre Formen des Kapitals durch die Einführung von Kapitalmarktinstrumenten wie z.B. Derivaten ablöst, ist die Intensivierung der Arbeit. Da das Kapital so eingesetzt wird, dass eine höhere Produktivität und Profitabilität erreicht wird, kommt der Arbeit nicht nur ein höheres Maß an Risiko zu, sie ist auch der Forderung nach erhöhter Produktivität, flexiblerer Zeiteinteilung und der Zahlung niedrigerer Reallöhne ausgeliefert. Dieser Zustand, der auch als Prekarität (oder als Abkehr von klassischen Vollzeitstellen und unbefristeten Arbeitsverhältnissen mit einem einzigen Arbeitgeber) bezeichnet wird, bringt die umgekehrte Proportionalität der extensiven und intensiven Momente der Arbeit in ein Ungleichgewicht (vgl. Neilson/Rossiter 2008; Ross 2009; Standing 2011). Eine wachsende Zahl prekärer Arbeiter ist nicht mehr in der Lage, einen Haushalt zu ernähren, und unter diesen Umständen wird die Fähigkeit der Arbeit, sich selbst zu reproduzieren, ungewiss. Arbeit ist deshalb zunehmend aufgeteilt zwischen denjenigen, die einen Haushalt ernähren können, und denjenigen, deren Fähigkeit, den eigenen Lebensunterhalt zu bestreiten, nicht bekannt oder höchst volatilen Nachfragebedingungen unterworfen ist. In jedem Fall aber gibt es eine Vervielfältigung der Arbeit, egal, ob es darum geht, den finanzialisierten Haushalt aufrechtzuerhalten (inklusive der Erhaltung des Körpers durch Training und Aktivitäten, die die Risikobelastung abschwächen) oder mit Jobs auf dem prekären Arbeitsmarkt zu jonglieren. Marazzi (2005, S. 111) beobachtet: „fixed capital, if it disappears in its material and fixed form, reappears in the mobile and fluid form of the living."

Die Intensivierung der Arbeit, die oben beschrieben wurde, vollzieht sich in den sogenannten fortgeschrittenen kapitalistischen westlichen Gesellschaften parallel zu Prozessen der Diversifizierung, die die Vormachtstellung eines besonders homogenen Typus - z.B. des Industriearbeiters - in der Gesamtheit der abhängigen Arbeit in Frage stellten.

Während Arbeit aufgrund einer intensiveren Kooperation und aufgrund der zunehmenden Bedeutung, die ,selbstverständlichen' Kompetenzen wie Wissen und Sprache zukommt, mehr und mehr durch soziale Merkmale bestimmt wird, werden einzelne Beschäftigungsverhältnisse sowohl im Hinblick auf Aufgaben und ,Fertigkeiten' als auch in Bezug auf rechtliche Bedingungen und Status vervielfältigt. Es ist nicht länger möglich, zu behaupten, dass die Arbeitsteilung zu mehr Solidarität und zu einem stärkeren Zusammenhalt innerhalb bestimmter Gruppen von Menschen in sozialen Einheiten führt, wie Emile Durkheim (1893) so eindrücklich Ende des 19. Jahrhunderts erörterte. Je mehr die Arbeit sozialisiert wird, desto fluider werden Beziehungen sozialer Solidarität. Statt anzunehmen, dass Gesellschaft ein Ganzes ist, das dann durch Arbeit geteilt wird, ist es notwendig, die Unterschiede, Inkonsistenzen und Multiplizitäten nachzuvollziehen, die sich des Feldes der Arbeit bemächtigen und im Gegenzug die organische Vorstellung von Gesellschaft zerlegen. Solch eine Heterogenisierung der Arbeit wird auch durch die Flexibilisierung des Arbeitsrechts und insbesondere durch die explosionsartige $\mathrm{Zu}$ nahme an Vertragsarrangements, die mit der Abnahme kollektiver Verhandlungen einhergeht, gespiegelt und verfestigt (vgl. Supiot 1994; 2001; Salento 2003). Sie wird auch erkennbar anhand der Zunahme von Kodizes und Chartas großer Unternehmen und Konzerne, die sich auf Arbeitsstandards und -bedingungen beziehen, insbesondere in Situationen, in denen der weltweite Bedarf des Kapitals an schlecht bezahlter Arbeit dafür sorgt, dass Verbraucherkampagnen und Kritik von Seiten der Politik drohen. Solche Kodizes und Standards weisen einen eindeutig performativen Charakter auf, können jedoch auch deutliche normative Tendenzen annehmen, die den Sektor des globalen Rechtswesens aufbrechen und zu einer Loslösung der Rechtsprechung von ihrem Geltungsbereich führen. 
Man kann diese Situation offensichtlich als weitere Vertiefung der Arbeitsteilung interpretieren, nun erweitert um die Kombination ihrer technischen und sozialen Dimension und um die Produktion einer neuen Anzahl von Grenzen über die Struktur der lebendigen Arbeit hinweg. Wir stellen dies nicht in Abrede. Indem wir den Aspekt der Vervielfältigung deutlicher hervorheben als den der Arbeitsteilung, möchten wir erstens auf die Unverhältnismäßigkeit zwischen der intensivierten sozialen Dimension der zeitgemäßen Arbeit („der Vereinigung von Kräften " [the conjunction of forces] im Sinne von Hume 1739/1973, S. 229) und der Vertiefung der sozialen und technischen Arbeitsteilung (der „Teilung der Arbeit“ [the partition of employments], um es erneut mit Hume [ebd.] zu sagen) hinweisen. Während Vervielfältigung auf diese Aspekte des strukturellen Exzesses verweist (die zeitgemäße Manifestation des „immanenten Widerspruchs“, der von Marx 1867/1986, Kap. 13, in seiner Analyse der „großen Industrie“ [ebd.] identifiziert wurde), zeigt sie auch das parallele Wirken der drei Tendenzen - Intensivierung, Diversifizierung und Heterogenisierung der Arbeit - auf, die zunehmend Arbeitserfahrungen und -bedingungen verändern. Die biopolitische Mobilisierung des Lebens, die aus der Kombination dieser Tendenzen resultiert, ermöglicht uns, die sich verändernde Struktur der lebendigen Arbeit im gegenwärtigen Kapitalismus zu interpretieren, ohne die großen Unterschiede zwischen dem Globalen Norden und dem Globalen Süden, den Kerngebieten und der Peripherie usw. zu berücksichtigen. Man könnte sogar sagen, dass die Peripherie in einer klassischen postkolonialen Bewegung zurückschlägt, weil die radikale Heterogenität der Arbeitsbeziehungen, die lange ein Charakteristikum der kolonialen Welt war, als Ergebnis der Prozesse, die wir oben skizziert haben, zunehmend die früheren Industrieregionen erfasst.

Noch einmal, es geht uns nicht darum, zu behaupten, dass Räume und Territorien keine wichtige Rolle mehr in der Struktur (sowie in der Teilung) von Arbeit spielen. Was wir über die vier aufstrebenden Branchen schreiben (vgl. Mezzadra/Neilson 2013, S. 86), die Beverly Silver (2003) identifiziert hat, trifft auch hier zu. Die Prozesse der Intensivierung, Diversifizierung und Heterogenisierung gestalten Arbeitsleben und -bedingungen über die unterschiedlichsten Räume und Reichweiten der globalen Operationen des Kapitals neu, aber sie bewirken sehr unterschiedliche konkrete Konstellationen aus Beschäftigung und Beschäftigungslosigkeit, aus Elend, Auskommen und Ausbeutung, aus Flucht, Ablehnung und Konflikten. Es ist sicherlich immer noch möglich, von einer globalen Arbeitsteilung zu sprechen, die Arbeiter innerhalb bestimmter Produktionszyklen und Gebrauchsgüterketten eint (und gleichermaßen voneinander trennt). Aber das Konzept der internationalen Arbeitsteilung wird aufgrund von Prozessen der Heterogenisierung, die nicht mehr Nationen, sondern eher Regionen als bedeutende wirtschaftliche Einheiten kennzeichnen, weniger relevant. Wenn wir dem Aspekt der Arbeitsteilung so viel Bedeutung beimessen, dann besteht die Gefahr, dass der Aspekt der Vervielfältigung, den wir hier beschrieben haben, allzu leicht in den Hintergrund rückt, ebenso wie die subjektiven Spannungen, Bewegungen und Konflikte, die damit einhergehen.

Während die sich ausweitenden Grenzen des Kapitals den Weltmarkt in die neue Dimension des globalen Finanzkapitalismus katapultiert haben, die das repräsentieren und umsetzen, was Deleuze und Guattari (1983) als „Axiomatik des Kapitals“ bezeichnen, ist die abstrakte Arbeit gewaltsam zum neuen Standard erhoben worden, dem das Leben auf dem gesamten Planeten unterworfen wird. Selbst die Bedarfsdeckungswirtschaft, von der die Reproduktion der großen Massen abhängt, beispielhaft von Mike Davis (2006) in Planet of Slums beschrieben, wird zunehmend in Finanzkreisläufe eingebunden. Die Verfügbarkeit von Mikrokrediten ist ein Instrument, durch das das gesamte Leben dieser Massen als Humankapital kodiert wird, das 
nicht verschwendet werden sollte (obwohl es häufig verschwendet wird), sondern stattdessen gezwungen wird, Werte nach der Logik der abstrakten Arbeit zu generieren. Aber die Allgegenwärtigkeit der abstrakten Arbeit schließt nicht die Lücke, die sie von der lebendigen Arbeit trennt (vgl. Chakrabarty 2000; Mezzadra 2011). Einerseits wird diese Lücke in den aktuellen Prozessen und Formen der Arbeit größer und in diesem Sinne entspricht diese Ausweitung dem Prinzip Teile und herrsche, andererseits hat die lebendige Arbeit immer noch die Chance, sich der Unterwerfung unter die Norm der abstrakten Arbeit zu verweigern - oder zumindest die Unterwerfung zur Verhandlungssache zu machen. Aus dieser Perspektive kann die Vervielfältigung der Arbeit zu einem unkalkulierbaren Element in den Beziehungen zwischen Kapital und Arbeit werden und zu unvorhersehbaren Spannungen, politischen Bewegungen und Konflikten führen. Routinen der Mobilität spielen eine wesentliche Rolle im Rahmen dieser Spannungen, politischen Bewegungen und Konflikte (wie sie es im Allgemeinen auch im Rahmen der aktuellen Prozesse der Vervielfältigung der Arbeit tun). Die Kontrolle über die Arbeitsmobilität ist auch eines der Schlüsselthemen, in denen die Lücken zwischen den sich ausweitenden Grenzen des Kapitals immer mehr von politischen und rechtlichen Grenzen durchdrungen werden. Hier spielt die Produktion der Arbeitskraft als Ware eine Schlüsselrolle und die Bedeutung, die der Grenze hinsichtlich der Gestaltung des Arbeitsmarktes zukommt (des Marktes, auf dem Arbeitskraft als Ware ausgetauscht wird), ist eine herausragende, aber häufig vernachlässigte Frage.

Aus dem Englischen von Ines Bergfort

\section{Literaturverzeichnis}

Balibar, Étienne (2002): Politics and the Other Scene. London: Verso.

Barth, Fredrik (1979): Ethnic Groups and Boundaries: The Social Organization of Cultural Difference. Oslo: Universitetsvorlaget.

Boltanski, Luc/Chiapello, Eve (2005): The New Spirit of Capitalism. New York: Verso.

Bryan, Dick/Martin, Randy/Rafferty, Mike (2009): Financialization and Marx: Giving Labor and Capital a Financial Makeover. In: Review of Radical Political Economics 41, H. 4, S. 58-72.

Cella, Gian Primo (2006): Tracciare confini: Realtà e metafore della distinzione. Bologna: Il Mulino.

Chakrabarty, Dipesh (2000): Provincializing Europe: Postcolonial Thought and Historical Difference. Princeton, NJ: Princeton University Press.

Cuttitta, Paolo (2007): Segnali di confine: Il controllo dell'immigrazione nel mondo-frontiera. Milan: Mimesis.

Davis, Mike (2006): Planet of Slums. London: Verso.

Deleuze, Gilles/Guattari, Félix (1983): Anti-Oedipus: Capitalism and Schizophrenia. Minneapolis: University of Minnesota Press.

Durkheim, Émile (1893): De la division du travail social. Paris: Félix Alcan.

Fumagalli, Andrea (2007): Bioeconomia e capitalismo cognitivo: Verso un nuovo paradigma di accumulazione. Rome: Carocci.

Hardt, Michael/Negri, Antonio (2004): Multitude: War and Democracy in the Age of Empire. New York: Penguin.

Hochschild, Arlie (1983): The Managed Heart: Commercialization of Human Feeling. Berkeley: University of California Press.

Hume, David (1739/1973): Traktat über die menschliche Natur. Hamburg: Felix Meiner.

Kern, Horst/Schumann, Michael (1984): Das Ende der Arbeitsteilung?: Rationalisierung in der industriellen Produktion: Bestandsaufnahme, Trendbestimmung. München: C. H. Beck.

Kolossov, Vladimir (2005): Border Studies: Changing Perspectives and Theoretical Approaches. In: Geopolitics 10, H. 4, S. 606-632.

Lamont, Michèle/Molnár, Virág (2002): The Study of Boundaries in the Social Sciences. In: Annual Review of Sociology 28, H. 1, S. 167-195. 
Law, John (2004): After Method: Mess in Social Science Research. London: Routledge.

Longo, Matthew (2017): The Politics of Borders. Cambridge: Cambridge University Press.

Marazzi, Christian (2005): Capitalismo digitale e modello antropogenetico del lavoro: L'ammortamento del corpo macchina. In: Laville, Jean Louis/Marazzi, Christian/La Rosa, Michele/Chicchi, Federico (Hrsg.): Reinventare il lavoro. Rome: Sapere 2000, S. 107-126.

Marazzi, Christian (2010): The Violence of Financial Capitalism. New York: Semiotext(e).

Marx, Karl (1857-1858/1967): Grundrisse der Kritik der politischen Ökonomie. (Rohentwurf). [Fotomechan. Nachdr. der Moskauer Ausg. von 1939 u. 1941]. Frankfurt/M.: Europäische Verlagsanstalt.

Marx, Karl (1867/1986): Das Kapital, Bd.1. Berlin: Dietz-Verlag.

Marx, Karl (1905-1910/1974): Theorien über den Mehrwert. Berlin: Dietz-Verlag.

Mercator, Gerardus (1595): Atlas sive Cosmographicae Meditationes de Fabrica Mundi et Fabricati Figura. Duisburg: Rumold Mercator.

Mezzadra, Sandro (2011): How Many Histories of Labour? Towards a Theory of Postcolonial Capitalism. In: Postcolonial Studies 14, H. 2, S. 151-170.

Mezzadra, Sandro/Neilson, Brett (2013): Border as Method, or, the Multiplication of Labor. Durham, London: Duke University Press.

Mezzadra, Sandro/Neilson, Brett (2019): The Politics of Operations. Excavating Contemporary Capitalism. Durham/London: Duke University Press.

Neilson, Brett/Rossiter, Ned (2008): Precarity as a Political Concept, or, Fordism as Exception. In: Theory, Culture and Society 25, H. 7-8, S. 51-72.

Newman, David/Paasi, Anssi (1998): Fences and Neighbors in the Postmodern World: Boundary Narratives in Political Geography. In: Progress in Human Geography 22, H. 2, S. 186-207.

Newman, David (2006): The Lines That Continue to Separate Us: Borders in Our „Borderless“ World. In: Progress in Human Geography 30, H. 2, S. 143-61.

Prescott, John Robert Victor (1987): Political Frontiers and Boundaries. London: Allen and Unwin.

Rodríguez, Nestor (1996): The Battle for the Border: Notes on Autonomous Migration, Transnational Communities and the State. In: Social Justice 23, H.3, S. 21-39.

Ross, Andrew (2009): Nice Work if You Can Get It: Life and Labor in Precarious Times. New York: New York University Press.

Salento, Franco Angeli (2003): Postfordismo e ideologie giuridiche: Nuove forme d'impresa e crisi del diritto del lavoro. Milan: Angeli.

Sassen, Saskia (2006): Territory, Authority, Rights: From Medieval to Global Assemblages. Princeton, NJ: Princeton University Press.

Sassen, Saskia (2007): A Sociology of Globalization. New York: W. W. Norton.

Schmitt, Carl (1950): Der Nomos der Erde im Völkerrecht des Jus Publicum Europaeum. Berlin: Duncker $\&$ Humblot.

Silver, Beverly (2003): Forces of Labor: Workers' Movements and Globalization since 1870. New York: Cambridge University Press.

Simmel, Georg (1908/2009): Sociology: Inquiries into the Construction of Social Forms. Leiden: Brill.

Smith, Adam (1776/2009): Der Wohlstand der Nationen. Köln: Anaconda.

Standing, Guy (2011): The Precariat: The New Dangerous Class. London: Bloomsbury Academic.

Supiot, Alain (1994): Critique du droit du travail. Paris: puf.

Supiot, Alain (2001): Beyond Employment: Changes in Work and the Future of Labour Law in Europe. New York: Oxford University Press.

Vila, Pablo (2000): Crossing Borders, Reinforcing Borders: Social Categories, Metaphors, and Narrative Identities on the U.S.-Mexico Frontier. Austin: University of Texas Press.

Virno, Paolo (2003): A Grammar of the Multitude: For an Analysis of Contemporary Forms of Life. Cambridge: Semiotext(e).

Wallerstein, Immanuel (1974): The Modern World-System: Capitalist Agriculture and the Origins of the European World-Economy in the Sixteenth Century. New York: Academic Press.

Weeks, Kathi (2007): Life within and against Work: Affective Labor, Feminist Critique, and Post-Fordist Politics. In: Ephemera: Theory \& Politics in Organization 7, H. 1, S. 233-249.

Zanini, Piero (1997): Significati del confine: I limiti naturali, storici, mentali. Milan: Bruno Mondadori. 